\title{
The Role of Statistical Models in Biometric Authentication
}

\author{
Sinjini Mitra ${ }^{1}$, Marios Savvides $^{2}$, and Anthony Brockwell ${ }^{1}$ \\ 1 Department of Statistics, \\ Carnegie Mellon University, Pittsburgh, PA 15213 \\ \{smitra, abrock\}@stat.cmu.edu \\ 2 Electrical and Computer Engineering Department, \\ Carnegie Mellon University, Pittsburgh, PA 15213 \\ msavvid@cs.cmu.edu
}

\begin{abstract}
The current paper demonstrates the role of statistical models in authentication tasks - both in system development and in performance evaluation. We first introduce a model-based face authentication system based on the Fourier domain phase using Gaussian Mixture Models (GMM) which yields verification error rates as low as $0.3 \%$ on a face database of 65 individuals with extreme illumination variations. We then present a statistical framework for predicting authentication error rates for future populations in a rigorous way. This is in contrast to most evaluation protocols used today that are based on observational studies and valid only for the databases at hand. Applications establish that our model-based approach has better predictive performance than an existing state-of-the-art authentication technique.
\end{abstract}

\section{Introduction}

There are two broad approaches to devising authentication systems: (1) featurebased, and (2) model-based. For facial biometrics, feature-based methods use individualized facial characteristics such as distance between eyes, nose, mouth, and their shapes and sizes as the matching criteria. The model-based systems, on the other hand, use a statistical model to represent the pattern of some facial features (often, the ones mentioned above), and then some characteristics of the fitted model, such as, parameters or likelihood, are used as the matching criteria. Feature-based methods are simple and less rigorous usually than the modelbased ones and often involve parameters that need to be chosen by extensive experimentation for a particular database.

Although the importance of models is well-understood and has been exploited quite extensively in image re-construction and segmentation, its use it devising face authentication systems has been relatively limited. Some common face models include Gaussian ([1]), Markov ([2], 3]). One class of flexible statistical models is the Gaussian Mixture Models (GMM; 4]), which represents complex distributions through an appropriate choice of its components to capture accurately the local areas of support of the true distribution. Apart from statistical 
applications, GMMs have also been used in computer vision for modeling the shape and texture of face images ([5]).

Most existing face recognition systems are based on spatial image intensities. Recently much research effort has focused on the frequency domain as well, whose useful properties have been successfully exploited in many signal processing applications ([6]). The frequency domain representation of an image consists of two components, the magnitude and phase. In $2 \mathrm{D}$ images particularly, the phase captures more of the image intelligibility than magnitude and hence is very significant for performing image reconstruction ([7]). 8] showed that correlation filters built in the frequency domain can be used for efficient face verification. Recently, 9 proposed correlation filters based only on phase which performed as well as the original filters, and [10] demonstrated that performing PCA in the frequency domain using only the phase spectrum outperforms spatial domain PCA and also has illumination tolerance. However, no face models have been developed, as per the authors' knowledge, in the frequency domain.

Another important component of biometric authentication is performance evaluation. Most face authentication systems are tested on small to moderatelysized databases, which is not adequate to address bigger questions about the expected performance on large-scale databases to which the system has not been previously exposed to. For example, say a certain system yields a false alarm rate of $1 \%$; this implies that a database of size $1,000,000$ will produce 10,000 false alarms and this is quite undesirable in practice. It is known that there are about 500 million border crossings per year in the United States (one-way only), so this system will result in a lot of innocent travelers being unnecessarily harassed and extra overhead (personnel, time) required to attend to them. Large-scale evaluation protocols used today are based on observational studies (like FRVT, 2002, 2004) but from a statistician's perspective, these are at most empirical in nature - there is no statistical basis (e.g.,modeling) and scope for valid inference. Our goal in this paper is to propose a framework for performing such large-scale inference based on statistical models which has the potential to be more reliable.

The paper is organized as follows. Section 2 gives a brief description of the database used and Section 3 presents our GMM-based authentication scheme. The statistical framework for performance evaluation is introduced in Section 4 and its application to our model-based scheme and comparison with an existing non model-based method appears in Section 5. Section 6 contains a discussion.

\section{Data}

We use a subset of the "CMU-PIE Database" (11) which contains frontal images of 65 people under 21 different illumination conditions from balanced to shadows. Images belonging to one person from the database appears in Figure 1] 


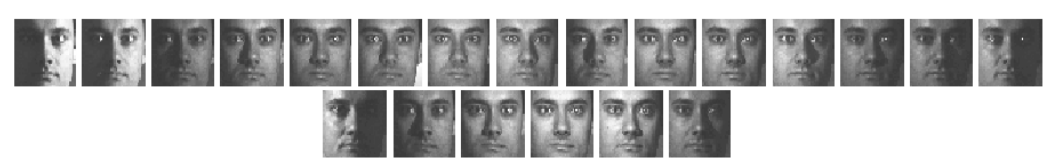

Fig. 1. Sample images of a person from the CMU-PIE database

\section{Model-Based System: GMM Based on Phase}

Mixture models provide a semiparametric framework for modeling unknown distributional shapes and can handle situations where a single parametric family is unable to provide a satisfactory model for local variations in the observed data.

Let $\left(\mathbf{Y}_{\mathbf{1}}, \ldots, \mathbf{Y}_{\mathbf{n}}\right)$ be a random sample of size $n$ where $\mathbf{Y}_{\mathbf{j}}$ is a $p$-dimensional random vector with probability distribution $f\left(\mathbf{y}_{\mathbf{j}}\right)$ on $\mathcal{R}^{p}$, and let $\boldsymbol{\theta}$ denote a vector of the model parameters to be estimated. A $g$-component mixture model can be written in parametric form as:

$$
f\left(\mathbf{y}_{\mathbf{j}} ; \Psi\right)=\sum_{i=1}^{g} \pi_{i} f_{i}\left(\mathbf{y}_{\mathbf{j}}, \boldsymbol{\theta}_{\boldsymbol{i}}\right),
$$

where $\boldsymbol{\Psi}=\left(\pi_{1}, \ldots, \pi_{g}, \boldsymbol{\theta}_{\mathbf{1}}, \ldots, \boldsymbol{\theta}_{\boldsymbol{g}}\right)^{T}$ contains the unknown parameters. Here, $\boldsymbol{\theta}_{\boldsymbol{i}}$ represents the model parameters for the $i^{t h}$ mixture component and $\boldsymbol{\pi}=$ $\left(\pi_{1}, \ldots, \pi_{g}\right)^{T}$ is the vector of the mixing proportions with $\sum_{i=1}^{g} \pi_{i}=1$. In case of Gaussian mixture models, the mixture components are multivariate Gaussian given by:

$$
\begin{aligned}
f\left(\mathbf{y}_{\mathbf{j}} ; \boldsymbol{\theta}_{\boldsymbol{i}}\right) & =\phi\left(\mathbf{y}_{\mathbf{j}} ; \boldsymbol{\mu}_{\boldsymbol{i}}, \boldsymbol{\Sigma}_{\mathbf{i}}\right) \\
& =(2 \pi)^{-1}\left|\boldsymbol{\Sigma}_{\mathbf{i}}\right|^{-\frac{1}{2}} \exp \left\{-\frac{1}{2}\left(\mathbf{y}_{\mathbf{j}}-\boldsymbol{\mu}_{\boldsymbol{i}}\right)^{T} \boldsymbol{\Sigma}_{\mathbf{i}}^{-1}\left(\mathbf{y}_{\mathbf{j}}-\boldsymbol{\mu}_{\boldsymbol{i}}\right)\right\}
\end{aligned}
$$

so that $\boldsymbol{\theta}_{\boldsymbol{i}}=\left(\boldsymbol{\mu}_{\boldsymbol{i}}, \boldsymbol{\Sigma}_{\mathbf{i}}\right), i=1, \ldots, g$ and the mixture model has the form:

$$
f\left(\mathbf{y}_{\mathbf{j}} ; \boldsymbol{\Psi}\right)=\sum_{i=1}^{g} \pi_{i} \phi\left(\mathbf{y}_{\mathbf{j}} ; \boldsymbol{\mu}_{\boldsymbol{i}}, \boldsymbol{\Sigma}_{\mathbf{i}}\right) .
$$

Despite the significance of phase in face identification tasks, modeling the phase angle poses several difficulties such as, the "wrapping around" property (it lies between $-\pi$ and $\pi$ ) and its sensitivity to distortions (such as illuminations). This prompted us to use the real and imaginary parts of the frequencies in the "phase-only" spectrum as an alternative representation of the phase. This is a simple yet effective way of modeling phase since it does not suffer from the difficulties associated with direct phase modeling.

Let $R_{s, t}^{k, j}$ and $I_{s, t}^{k, j}$ respectively denote the real and the imaginary parts at frequency $(s, t)$ of the phase spectrum of the $j^{t h}$ image from the $k^{t h}$ person, $s, t=1,2, \ldots, k=1, \ldots, 65, j=1, \ldots, 21$. We model $\left(R_{s, t}^{k, j}, I_{s, t}^{k, j}\right), j=1, \ldots, 21$ as a mixture of bivariate Gaussians whose density is given by Eqn.(3) for each 
$(s, t)$ and each person $k$. We model only the low frequencies within a $50 \times 50$ grid around the spectrum origin since they capture all the image identifiability ([6]), thus achieving considerable dimension reduction. We use a Gibbs sampler for estimating the unknown parameters $\boldsymbol{\Psi}$ using posterior means ([12]).

\subsection{Classification and Verification}

Classification of a new test image is done with the help of a MAP (maximum a posteriori) estimate based on the posterior likelihood of the data. For a new observation $Y=\left(R^{j}, I^{j}\right)$ extracted from the phase spectrum of a new image, it is assigned to class $C$ if

$$
C=\arg \max _{k} f(k \mid Y),
$$

where $f(k \mid Y) \propto g(Y \mid k) p(k)$ with $g(Y \mid k)=\Pi_{s} \Pi_{t} f_{k}^{s, t}\left(\mathbf{y}_{\mathbf{j}} ; \boldsymbol{\Psi}\right)$ assuming independence among frequencies and uniform priors over all the people and where $f_{k}^{s, t}\left(\mathbf{y}_{\mathbf{j}} ; \boldsymbol{\Psi}\right)$ denotes the likelihood of $Y$ for person $k$ at frequency $(s, t)$.

Table 1 shows the classification results for our database using different number of training images and $g=2$. The training set in each case is randomly selected and the rest are used for testing. This selection of the training set is repeated 20 times (in order to remove selection bias) and the final errors are obtained by averaging over those from the 20 iterations. The results are fairly good,

Table 1. Identification error rates for GMM-based system $(g=2)$

\begin{tabular}{|c|c||c|c|}
\hline \# of Training images & \# of test images & Error Rates & Std. dev. over 20 repetitions \\
\hline 15 & 6 & $1.25 \%$ & $0.69 \%$ \\
10 & 11 & $2.25 \%$ & $1.12 \%$ \\
6 & 15 & $9.67 \%$ & $2.89 \%$ \\
\hline
\end{tabular}

which demonstrates that our model is able to capture the illumination variation suitably. However, an adequate number of training images is required for the efficient estimation of the parameters; in our case, 10 is the optimal number of training images required. The associated standard errors in each case also prove the consistency of the results. Increasing the number of mixture components ( $g=3$ and $g=4$ ) do not improve results significantly; hence a 2-component GMM represents the best parsimonious model in this case.

Verification is performed by imposing a threshold on the log-likelihood of the test images. Satisfactory results are achieved with the optimal model $(g=2$ and 10 training images) that yields an Equal Error Rate (EER) of $0.3 \%$ at a threshold value of -1700 (ROC curve not included for space constraints).

\section{Large-Scale Performance: Random Effects Model}

Traditional performance evaluation tools like linear regression models, ANOVA ([13]) are fixed effects models and the inference from them cannot be generalized beyond the people in the database at hand. This difficulty can be obviated 
by the use of random effects models ([12]) which provide a flexible framework for extending inference to a bigger population by assuming that the particular subset of subjects in the current database is a random sample from a bigger population. The regression framework allows the inclusion of any number of potential covariates representing image properties (distortions, clarity) and system design parameters which may influence the system performance, and quantitatively determine which of these factors significantly affect performance in a general population and to what extent. They are thus crucial for large-scale inference and also take into account the heterogeneity across individuals in their regression coefficients with the help of a probability distribution. Some particular questions of interest in this context are:

- What are the effects of certain image properties and system parameters on the score distribution in the population?

- What is the predicted score distribution for authentics and impostors and error rates for an unknown large population?

\subsection{The Model Framework}

Let $Y_{i j}$ denote the outcome for the $j^{\text {th }}$ observation on the $i^{\text {th }}$ subject in the database, while $x_{i j}^{(m)}$ denotes the corresponding value for covariate $m$. We adopt the following hierarchical model:

$$
Y_{i j} \stackrel{i n d .}{\sim} N\left(\alpha_{i}+\sum_{m=1}^{M} \beta_{i}^{m} x_{i j}^{(m)}, \sigma^{2}\right), \quad i=1, \ldots, k, \quad j=1, \ldots, n_{i},
$$

where $M$ is the total number of covariates in the study. We assume that the slope-intercept vectors for each individual are drawn from a common population:

$$
\boldsymbol{\theta}_{\boldsymbol{i}} \equiv\left(\alpha_{i}, \beta_{i}^{1}, \ldots, \beta_{i}^{M}\right)^{T} \sim M V N\left(\boldsymbol{\theta}_{\mathbf{0}} \equiv\left(\alpha_{0}, \beta_{0}^{1}, \ldots, \beta_{0}^{M}\right)^{T}, \Sigma\right), i=1, \ldots, k
$$

We then select conjugate hyperpriors for the other parameters as follows:

$$
\sigma^{2} \sim I G(a, b), \quad \boldsymbol{\theta}_{\mathbf{0}} \sim N(\boldsymbol{\eta}, C), \quad \Sigma^{-1} \sim \operatorname{Wishart}\left((\rho R)^{-1}, \rho\right),
$$

where $R$ is a matrix and $\rho \geq 2$ is a scalar "degrees of freedom" parameter. The hyperparameters in the model $a, b, \boldsymbol{\eta}, C, \rho, R$ are assumed known. The unknown parameters are then estimated by using a Gibbs sampler by simulating from the respective full conditionals, assumed independence, which are:

$$
\begin{gathered}
\boldsymbol{\theta}_{\boldsymbol{i}} \mid \mathbf{y}, \boldsymbol{\theta}_{\mathbf{0}}, \Sigma^{-1}, \sigma^{2} \sim N\left(D_{i}\left(\frac{1}{\sigma^{2}} X_{i}^{T} \mathbf{y}_{\mathbf{i}}+\Sigma^{-1} \boldsymbol{\theta}_{\mathbf{0}}\right), D_{i}\right), i=1, \ldots, k, \text { where } \\
D_{i}^{-1}=\frac{1}{\sigma^{2}} X_{i}^{T} X_{i}+\Sigma^{-1}, \quad \mathbf{y}_{\mathbf{i}}, X_{i}=\text { variables for person i. } \\
\boldsymbol{\theta}_{\mathbf{0}} \mid \mathbf{y}, \boldsymbol{\theta}_{\boldsymbol{i}}, \Sigma^{-1}, \sigma^{2} \sim N\left(V\left(k \Sigma^{-1} \overline{\boldsymbol{\theta}}+C^{-1} \boldsymbol{\eta}\right), V\right), \text { where } V=\left(k \Sigma^{-1}+C^{-1}\right)^{-1}, \overline{\boldsymbol{\theta}}=\frac{1}{k} \sum_{i=1}^{k} \boldsymbol{\theta}_{\boldsymbol{i}} .
\end{gathered}
$$




$$
\begin{gathered}
\Sigma^{-1} \mid \mathbf{y}, \boldsymbol{\theta}_{\boldsymbol{i}}, \boldsymbol{\theta}_{\mathbf{0}}, \sigma^{2} \sim \text { Wishart }\left(\left[\sum_{i=1}^{k}\left(\boldsymbol{\theta}_{\boldsymbol{i}}-\boldsymbol{\theta}_{\mathbf{0}}\right)\left(\boldsymbol{\theta}_{\boldsymbol{i}}-\boldsymbol{\theta}_{\mathbf{0}}\right)^{T}+\rho R\right]^{-1}, k+\rho\right) . \\
\sigma^{2} \mid \mathbf{y}, \boldsymbol{\theta}_{\boldsymbol{i}}, \boldsymbol{\theta}_{\mathbf{0}}, \Sigma^{-1} \sim I G\left(\frac{n}{2}+a, \frac{1}{2} \sum_{i=1}^{k}\left(\mathbf{y}_{\mathbf{i}}-X_{i} \boldsymbol{\theta}_{\boldsymbol{i}}\right)^{T}\left(\mathbf{y}_{\mathbf{i}}-X_{i} \boldsymbol{\theta}_{\boldsymbol{i}}\right)+b\right), n=\sum_{i=1}^{k} n_{i} .
\end{gathered}
$$

Inference from this model is based on the marginal posteriors for the population parameters $\boldsymbol{\theta}_{\mathbf{0}}=\left(\alpha_{0}, \beta_{0}^{1}, \ldots, \beta_{0}^{M}\right)$ and posterior predictive distributions $p\left(y_{i j} \mid \mathbf{y}\right)$. This is obtained with Gaussian kernel density estimates for new data generated using Equation 5 with the post-convergence values of the parameters.

\section{Application}

We apply the random effects methodology to the GMM-based system and to an existing system called the Minimum Energy Correlation Filter (MACE; 8]) using their authentication results on the PIE database. The response variable in each case is the authentication score - log-likelihood for GMM and log(PSR) for MACE (PSR = Peak-to-Sidelobe Ratio; 8]). We use a covariate denoting authenticity for both systems, but add one representing the illumination effect in MACE (since GMM modeled this). We again use a Gibbs sampler for parameter estimation and posterior means as the estimates. Table 2 shows the estimates of the population parameter $\boldsymbol{\theta}_{\mathbf{0}}$ which quantitatively show the effect of the covariates on the scores. We see that authenticity has a significant effect on authentication score for both systems while illumination has no effect on PSR.

The posterior predictive distributions of the authentication scores for the two systems are shown in Figure 2. We generated 1000 values for each system and 500 each for the authentics and the impostors. As can be seen, there exists a clear separation among the predicted values of authentic and impostor people; in fact, the distributions of the score statistics appear to be a mixture of two distinct distributions. The amount of overlap in the tails of the authentic and impostor distributions indicates the chances of false alarm and this clearly shows that the GMM method has a much reduced risk than the MACE system.

We next estimate the predicted FAR and FRR using closed-form expressions based on Gaussian kernels as functions of the authentication threshold $\tau$. Let $T$ denote the PSR values, and $f_{A}(\cdot)$ and $g_{I}(\cdot)$ respectively be the posterior

Table 2. Estimates of $\boldsymbol{\theta}_{\mathbf{0}}$ and $95 \%$ predictive intervals for the two systems

\begin{tabular}{|c||c|c|c|c|}
\hline System & Parameter & Estimate & Lower 95\% CI & Upper 95\% CI \\
\hline MACE & $\alpha$ & 1.9737 & 0.7504 & 3.1971 \\
& $\beta_{0}$ & 1.4634 & 1.2874 & 1.6395 \\
& $\gamma_{0}$ & -0.0184 & -0.1965 & 0.1581 \\
\hline GMM-based & $\alpha_{0}$ & -1694.4 & -1698.0 & -1690.8 \\
& $\beta_{0}$ & 81.7 & 76.7 & 86.7 \\
\hline
\end{tabular}




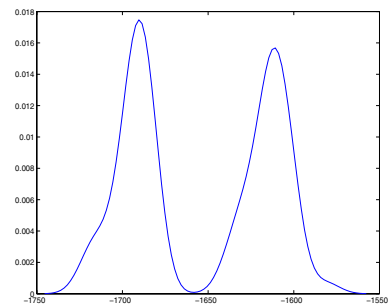

(a) GMM (log-likelihood)

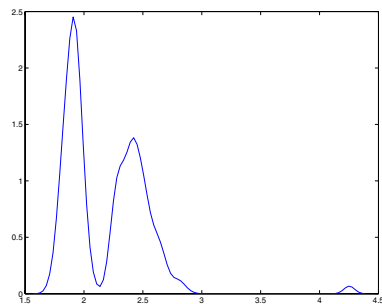

(b) MACE $(\log (\mathrm{PSR}))$

Fig. 2. Predictive posterior distribution of scores for the two systems

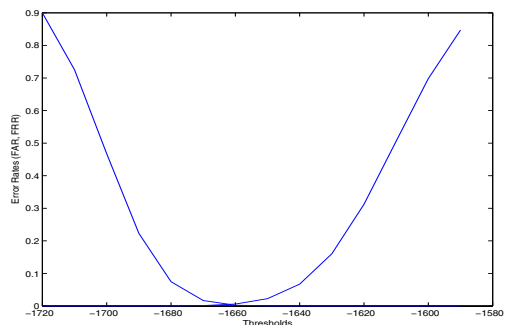

(a) $\operatorname{GMM}(\mathrm{EER}=0.8 \%)$

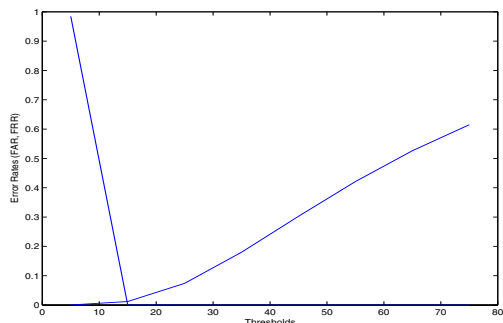

(b) $\mathrm{MACE}(\mathrm{EER}=1.5 \%)$

Fig. 3. Predicted false alarm rates for the two systems. The decreasing curve is the FAR, and the increasing one is the FRR.

predictive distributions of $\log (T)$ for the authentics and the impostors. If $\tau$ is the given threshold for authentication, the FAR and FRR are defined as:

$$
\begin{gathered}
F R R=P(T \leq \tau \mid \text { Authentic })=P(\log (T) \leq \log (\tau) \mid \text { Authentic })=\int_{-\infty}^{\tau} f_{A}(x) d x \\
F A R=P(T>\tau \mid \text { Impostor })=P(\log (T)>\log (\tau) \mid \text { Impostor })=\int_{\tau}^{\infty} g_{I}(y) d(y 9)
\end{gathered}
$$

Now if $f_{A}$ and $g_{I}$ be Gaussian with means $\left(\mu_{A}, \nu_{I}\right)$ and variances $\left(\sigma_{A}^{2}, \eta_{I}^{2}\right)$, these can be written in a in terms of $\Phi$ (distribution function of standard normal) as:

$$
F R R=\Phi\left(\frac{\log (\tau)-\mu_{A}}{\sigma_{A}}\right), \quad F A R=1-\Phi\left(\frac{\log (\tau)-\nu_{I}}{\eta_{I}}\right) .
$$

The resulting FAR and FRR for the two systems are shown in Figure 3 . The predicted EERs are $0.8 \%$ for the GMM system at a threshold log-likelihood value of -1650 and $1.5 \%$ for MACE at a threshold PSR value of 15 .

\section{Discussion}

This paper presented a face authentication scheme based on phase and GMM. Although the importance of phase is well-known, this fact had not been utilized in building model-based classification techniques. This is partially because modeling phase variations is a challenging task and our results show convincingly 
that the proposed model is able to handle it perfectly. In fact, we believe that owing to its general framework, our model should easily be applied to other distortions as well, such as, expression, noise, pose, by assigning different types of images to different components of mixture distributions. This proves the practical utility of this method for handling real life databases that are often subject to extraneous variations. In conclusion, harnessing the combined potential of GMM and phase has indeed proved to be a grand success.

We then proposed a novel statistical framework based on random effects model to predict the performance of a biometric system on unknown large databases. We applied this to the MACE system and our GMM based system, and established that the latter has a superior performance in terms of predictive performance. Development of such a rigorous evaluation protocol is feasible only with the help of statistical models which helps assess the true potential of authentication systems in handling real-world applications. This is the first of its kind and hence replaces the empirical and naive approaches based on observational studies that were being used until now. It is fairly general and easily extends to other biometrics as well. In conclusion, both our techniques have established the significant role played by statistical modeling tools in the technology of biometric authentication.

\section{References}

1. Turk, M.A., Pentland, A.P.: Face recognition using eigenfaces. In Proceedings of CVPR (1991)

2. Yuille, A. (1991): Deformable templates for face recognition. Journal of Cognitive Neuroscience 3 (1991)

3. Liu, C., Zhu, S.C., Shum, H.Y.: Learning inhomogeneous gibbs model of faces by minimax entropy. In Proceedings of ICCV (2001) 281-287

4. McLachlan, G., Peel, D.: Finite Mixture Models. John Wiley and Sons (2000)

5. Zhu, S., Wu, Y., Mumford, D.: Minimax entropy principle and its application to texture modeling. Neural Computation 9 (1997)

6. Oppenheim, A.V., Schafer, R.W.: Discrete-time Signal Processing. Prentice Hall, NJ (1989)

7. Hayes, M.H.: The reconstruction of a multidimensional sequence from the phase or magnitude of its fourier transform. ASSP 30 (1982) 140-154

8. Savvides, M., Vijaya Kumar, B.V.K., Khosla, P.: Face verification using correlation filters. In: 3rd IEEE Automatic Identification Advanced Technologies, Tarrytown, NY (2002) 56-61

9. Savvides, M., Kumar, B.V.K.: Eigenphases vs.eigenfaces. In Proceedings of ICPR (2004)

10. Savvides, M., Kumar, B.V.K., Khosla, P.K.: Corefaces - robust shift invariant PCA based correlation filter for illumination tolerant face recognition. CVPR (2004)

11. Sim, T., Baker, S., Bsat, M.: The CMU pose, illumination, and expression (PIE) database. In: Proceedings of the 5th International Conference on Automatic Face and Gesture Recognition. (2002)

12. Gelfand, A.E., Hills, S.E., Racine-Poon, A., Smith,A.F.M.: Illustration of bayesian inference in normal data models using gibbs sampling. Journal of the American Statistical Association 85 (1990) 972-985

13. Weisberg, S.: Applied Linear Regression. Wiley (1985) 